\title{
MULTIPLE LYMPHATIC-VENOUS ANASTOMOSES (MILVA) FOR MICROSURGICAL DRAINAGE OF PRIMARY PENO-SCROTAL LYMIPHEDEMA: A CASE REPORT
}

\author{
P.G. di Summa, M. Guiotto, P. Zaugg, A. Van Der Gucht, M. Ryan, \\ C.C. Campisi, W. Raffoul
}

Departments of Plastic, Reconstructive and Hand Surgery (PgdS,MG,PZ,WR) and Nuclear Medicine (AVDG), Centre Hospitalier Universitaire Vaudois (CHUV), Lausanne, Switzerland; Plastic, Reconstructive and Aesthetic Surgery, Lymphatic Surgery and Microsurgery ICLAS (Rapallo, Genoa), Salus Hospital (Reggio Emilia), Maria Pia Hospital (Turin) GVM Care \& Research (MR,CCC), Italy

\section{ABSTRACT}

Single site Multiple LymphaticVenous Anastomoses (MLVA) provides optimal functional and cosmetic results with low complication rates in treatment of lower limb lymphedema. However, no evidence exists in literature concerning the use of this technique in treatment of peno-scrotal lymphedema. We report a case of a 44-year-old male patient who developed secondary peno-scrotal lymphedema with severe lymphorrhea, following a laser treatment for scrotal pustolosis, leading to recurrent infections and finally an established peno-scrotal lymphedema. Utilizing MLVA, a complete remission of scrotal lymphedema was achieved with significant volume reduction of the penile lymphedema. The postoperative course was uneventful with clear improvement in lymphatic flow demonstrable on lymphoscintigraphy (6 months) and no recurrence of scrotal lymphedema at 2 years follow-up. This article reports very promising results of a novel application of MLVA in the treatment of genital lymphedema and suggests that MLVA provides the possibility to shunt both superficial and deep lymphatics to improve the lymphatic drainage from the peno-scrotal area using a single surgical site.
Keywords: microsurgery, lymphatic surgery, Multiple Lymphatic-Venous Anastomoses, peno-scrotal lymphedema

Genital lymphedema is a chronic and progressive disease with abnormal retention of fluid in subcutaneous tissues resulting in an increased volume of the external genital organs due to inadequate lymph drainage. Clinically it is characterized by heaviness, pain, recurrent infections, lymphorrhea, and urinary and sexual disorders, significantly compromising the quality of life (1).

The lymphatic drainage of the male genitalia is through a diffuse lymphatic network organized into two lymphatic plexuses: one from the testicular parenchyma and a second one from the tunica vaginalis and scrotal interstitial tissue. The testicular parenchymal lymphatics run proximally through the spermatic cord along the testicular artery to reach the para- and pre-aortic lymph nodes and partially via deep inguinal nodes to the external iliac nodes. The scrotal and tunica vaginalis lymphatics run through the superficial inguinal to the external iliac nodes. Concerning the penis shaft, the skin also drains to the superficial inguinal nodes while the penile body (glans) drains to the deep inguinal system (2).

Despite recently renewed interest in 
lymphedema surgery, there is a paucity of literature about surgical options for genital lymphedema. Surgical options can be either palliative or functional. Palliative options mainly involve radical excision of affected tissue (lymphangiectomy) followed by reconstruction of the genital area by local flaps or skin grafting (3). Functional treatments aim to re-establish or improve lymphatic flow. Developments in microsurgery have made lymphatic-venous anastomosis (LVA) an alternative surgical method for lymphedema treatment utilizing improved lymphatic drainage by shunting lymph into the venous bloodstream (1).

Conveying multiple lymphatics into a superficial vein (MLVA) at the inguinal level follows the lymphatic-venous anastomoses concept of shunting lymph into the venous system and bypassing an obstruction or an area where the lymphatic network is no longer competent. The MLVA technique, however, differs from the general LVA approach by using multiple lymphatic vessels and directing them into a single vein (high output unidirectional lymphovenous drainage) (4) without need for supermicrosurgical instruments and several anastomotic sites.

A key advantage of the inguinal region is that lymphatic-venous shunts can be either from residual superficial or the deep lymphatic systems which are normally of greater caliber and generally less affected by the degeneration process associated with chronic lymphedema (1).

Despite the fact that MLVA techniques have been routinely used for lower limb lymphedema (4), no specific data regarding MLVA for genital lymphedema treatment have been reported. Although a limited number of microsurgeons have performed LVA in this area, none concerning genital lymphedema have been reported. To our knowledge we describe the first case of peno-scrotal lymphedema treated with MLVA procedure with satisfactory cosmetic and functional outcomes.

\section{CASE REPORT}

A 44-year-old male presented with previous history of cryptorchidism treated at the age of 12 years and circumcision at the age of 17 years. At the age of 37, he developed skin infections with progressive scrotal pustolosis that was treated by laser and resulted in development of secondary peno-scrotal lymphedema in the following years. When admitted to our clinic, the lymphedema had been clinically evident for 12 months with active lymphorrhea, recurrent infections, and severe compromise of his professional and private life (Fig. 1A). The genital lymphedema was associated with a feeling of heaviness of the left lower limb. Venous ultrasound demonstrated effective venous function with continence of the great saphenous vein in both limbs. The patient underwent a preoperative genital lymphoscintigraphy (Fig. 2A). Also, a whole-body scan was performed to permit anatomic localization of the areas visualized. Images showed abnormal peno-scrotal lymph stasis of the radiotracer and non-visualization of the left inguinal/ iliac lymph node (Fig. 3A).

For the MLVA surgery, the patient was placed supine under general anaesthesia. On-table ICG injection was performed at the scrotal and penis shaft without identifying a clear pattern of superficial lymphatic flow. Injection of blue patent dye (Bleu Patente V sol inj., Guerbet, France) was performed at the peno-scrotal level (medial, right, left).

After incision at the groin level, we could identify, just under the level of Scarpa's fascia, a visibly sclerotic medial lymph node particularly adherent to deep planes with three afferent lymphatics. Two of these were carrying blue dye demonstrating their patency and proving no involvement in the sclerotic processes. After establishing the lymphatic block at the level of the sclerotic node, further injections of blue dye were performed at the suprainguinal level and on the medial thigh to identify supplementary lymphatic pathways.

The superficial inferior epigastric vein was followed cranially and divided allowing 

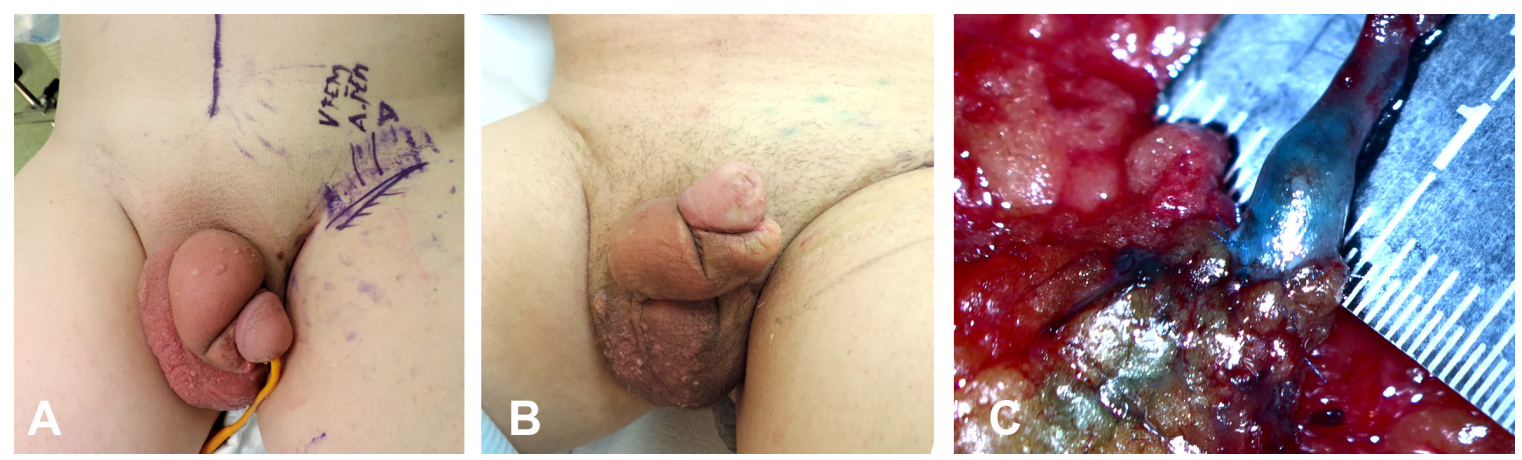

Fig. 1. A 44-year-old male patient with primary peno-scrotal lymphedema and active lymphorrhea, A: pre-operative clinical evaluation B: 6 months post-operative clinical evaluation with reduction of scrotal lymphedema, and significant amelioration of penile body lymphedema, C: MLVA performed to the SIEV. Afferent lymphatics were pulled inside the competent vein by a $U$ stitch, while perilymphatic tissue is secured circumferentially to the vein, as described by Campisi et al (8) with verification of the patency of lymphatic venous anastomosis under the operating microscope
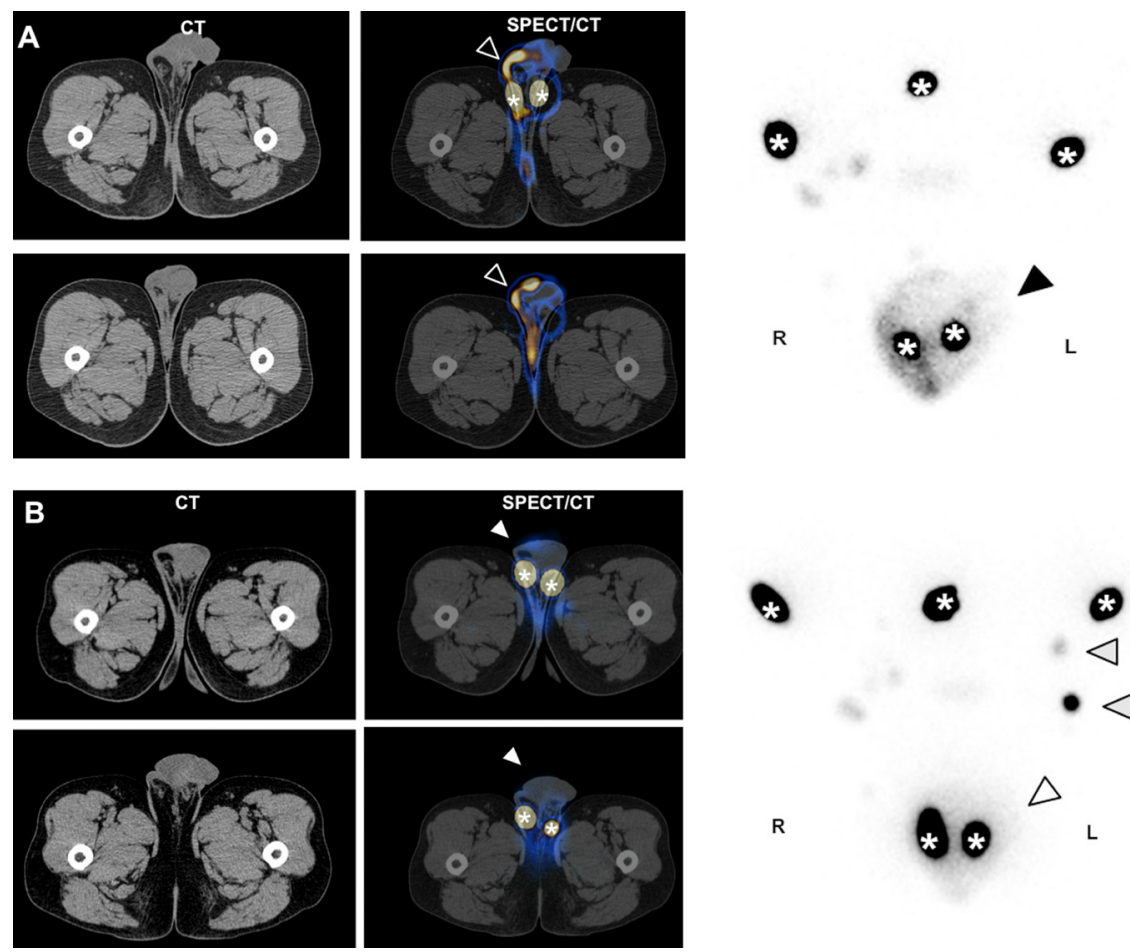

Fig. 2. Genital lymphoscintigraphy using a dual-head gamma camera system (Discovery NM/CT 670, GE Healthcare, USA). Asterisks indicate the 5 points of subcutaneous injections (2 in scrotal areas, 2 in inguinal areas and 1 subumbilical) of $99 \mathrm{mTc}$-filtered sulfur colloid (20 MBq per injection). A dynamic protocol over 15 min was used to acquire the images and also static images were taken three hours following injections. A: pre-operative functional evaluation showed abnormal peno-scrotal lymph stasis of the radiotracer (black arrowheads) and no left inguinall iliac lymph node was visualized (images acquired by a dynamic protocol over 15 min and static images taken three hours following injections). B: a 6 month follow-up functional evaluation showed a decreased uptake of the radiotracer on the scintigraphic acquisition (white arrowheads), confirmed the peno-scrotal lymph stasis reduction and amelioration of the left inguinal/iliac lymphatic drainage with visualization of lymph nodes (grey arrowheads). 
A

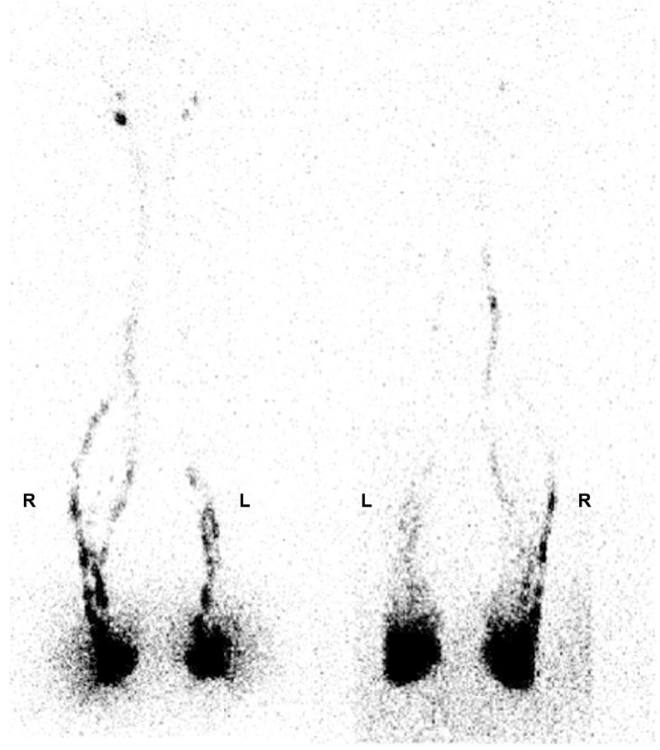

B

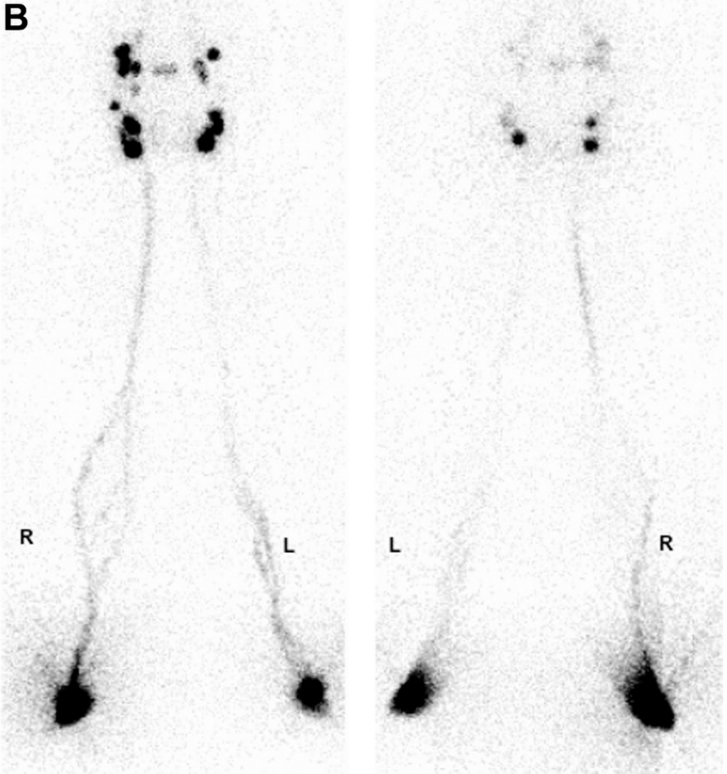

Fig. 3. Genital and lower limbs lymphoscintigraphy using a dual-head gamma camera system (Discovery NM/CT 670, GE Healthcare, USA) A: pre-operative evaluation front to back and in the opposite direction B: 6 month postoperative evaluation front to back and in the opposite direction

enough length to perform the MLVA. The vein was flushed with heparinized saline and checked for competence. All three lymphatic vessels were directly introduced together into the vein by a temporary U-shaped stitch and then fixed to the vein cut-end by means of multiple stitches between the vein border and the perilymphatic adipose tissue. Passage of the blue lymph into the vein branch allowed verification of patency of the lymphatic-venous anastomosis under the operating microscope (Fig. 1C). After accurate hemostasis, the wound was closed with monocryl 3.0 (Ethicon Inc, Johnson \& Johnson, Georgia, USA) in two layers over a Blake suction drain. Recovery was uneventful. Drains were removed at day two (when patient started mobilization) and the patient was discharged at day three.

Lymphorrhea ceased immediately postoperatively and significant amelioration of penile body lymphedema was evident at 6 months post-op (Fig. 1B). The patient was able to restart work activity at $80 \%$ (4/5 days a week), which required standing for 10 hours/ day, without any increase in penile or scrotal volume.

Genital lymphoscintigraphy at 6 months follow-up confirmed significant peno-scrotal lymph stasis reduction with a decreased uptake of the radiotracer (Fig. $2 B$ - white arrowheads) indicating transit into the vein via the new MLVA anastomoses as well as amelioration of the left inguinal/iliac lymphatic drainage with visualization of lymph nodes (Fig. $2 B$ - grey arrowheads). Lymphoscintigraphic evidence supports the clinical findings by demonstrating MLVA use to bypass a sclerotic lymph node blockage, enhance the lymphatic drainage of the genitalia, and reduce lymph stasis (Fig. 3B). No clinical recurrences of lymphedema were seen at 2 years follow-up.

\section{DISCUSSION}

Genital lymphedema can be a primary condition that severely compromises the qual- 
ity of life with genital swelling, lymphorrhea, pain, and infections. Late diagnosis, often due to embarrassment, means treatment begins at an advanced stage frequently with extensive fibrosis and damage to the lymphatic system (5).

For lower limb lymphedema, LVAs are considered the first choice among microsurgical treatments in earlier stages (I or II) after failure of conservative treatment. Recently, Scaglioni et al presented a case of combined genital and lower limb lymphedema where multi-site LVAs at the level of the ankle, distal lower leg, and lateral knee reduced lower limb swelling but did not affect genital lymphedema, and four months after the microsurgical procedure, a debulking of the excessive peno-scrotal mass was undertaken to finally improve symptoms at the genital site (6). Mukenge et al reported successfully treating advanced stage penile lymphedema with anastomoses from lymphatic vessels in the spermatic cord (afferent to the external iliac chains) to the veins of the pampiniform plexus, which are conveniently located within the spermatic cord adjacent to lymphatics (7).

Multiple Lymphatic-Venous Anastomoses (MLVA) represents a single-site microsurgical technique where multiple lymphatics converge into a single vein with a competent valve.

It can be applied both to the superficial and deep inguinal lymph node lymphatics, which is different from the general LVA techniques used by other authors (4). At the groin, both the superficial and deep inguinal lymphatic system can be directed to nearby veins with the possibility to separately drain the lymphatics afferent to either superficial and/or deep nodes. Previous use of MLVA on deep lymphatics, with a superficial network compromised, has shown to be beneficial because these are of greater caliber and generally less affected by the degeneration process (8).

To our knowledge, we describe the first case of peno-scrotal lymphedema treated with MLVA procedure with satisfactory cosmetic and functional outcomes. In this case, blue patent dye selective injection of the scrotum identified lymphatics to be targeted for the anastomoses. Visualization of the blue dye within the functioning lymphatics converging towards a clinically adherent and sclerotic inguinal node, blocking scrotal outflow, guided the surgical choice. Anatomical studies on Vascularized Lymph Node Transfer (VLNT) (2) have shown that lymph nodes of the superficial medial thigh that receive the superficial lymph flow are partially connected to the deep inguinal lymph node system thereby draining part of the deep component before merging into the external iliac lymph chain. In this sense, deriving afferent lymphatics to such nodes for the MLVA surgery at the groin may have beneficial effects on both lymphatic systems providing effective results.

Postoperative lymphoscintigraphy matched the clinical outcome showing a local decreased uptake of the radiotracer due to the functioning MLVA anastomoses directing the lymph to the bloodstream and amelioration of the left inguinal/iliac lymphatic drainage with visualization of an active femoral node, which was not clearly visible at the same exposure time in preoperative lymphoscintigraphy.

Genital lymphedema represents a challenge for microsurgeons and often debulking procedures represent the only surgical choice to achieve a significant amelioration of symptoms, especially in advanced stages. However, by considering the possibility to perform derivative lymphatic-venous shunts at the level of both the superficial and deep lymphatic system with the MLVA technique, even patients with more advanced stage lymphedema could also have clinical and functional ameliorations $(1,7,9)$. The MLVA technique, which has been extensively used in lower limb lymphedema, has low morbidity and low complication rates and it is relatively minimally invasive by comparison to excisional procedures, which could be considered as an always-available second option in case of unsatisfactory outcomes. 
CONFLICT OF INTEREST AND DISCLOSURE

The authors declare no competing financial interests exist.

\section{REFERENCES}

1. Mukenge, SM, M Catena, D Negrini, et al: Assessment and follow-up of patency after lymphovenous microsurgery for treatment of secondary lymphoedema in external male genital organs. Eur. Urology 60 (2011), 1114-1119.

2. Scaglioni, MF, H Suami: Lymphatic anatomy of the inguinal region in aid of vascularized lymph node flap harvesting. J. Plas. Recon. \& Aesth. Surg. 68 (2015), 419-427.

3. Guiotto, M, RJ Bramhall, CC Campisi, et al: A systematic review of outcomes after genital lymphedema surgery: Microsurgical reconstruction versus excisional procedures. Ann. Plast. Surg. (2019). May 22. doi: 10.1097/ SAP.0000000000001875. [Epub ahead of print]

4. Boccardo, F, S Dessalvi, C Campisi, et al: Microsurgery for groin lymphocele and lymphoedema after oncologic surgery. Microsurgery 34 (2014), 10-13.

5. Singh, V, RJ Sinha, SN Sankhwar, et al: Reconstructive surgery for penoscrotal filarial lymphoedema: A decade of experience and follow-up. Urology 77 (2011), 1228-1231.
6. Scaglioni, MF, S Uyulmaz: Lymphovenous anastomosis and debulking procedure for treatment of combined severe lower extremity and genital lymphoedema: A case report. Microsurgery 38 (2018), 907-911.

7. Mukenge, S, C Pulitano, R Colombo, et al: Secondary scrotal lymphoedema: A novel microsurgical approach. Microsurgery 27 (2007), 655-656.

8. Campisi, CC, M Ryan, F Boccardo, et al: A single-site technique of multiple lymphatic-venous anastomoses for the treatment of peripheral lymphoedema: Long-term clinical outcome. J. Recon. Microsurg. 32 (2016), 4249.

9. Yamamoto, T, I Koshima, H Yoshimatsu, et al: Simultaneous multi-site lymphaticovenular anastomoses for primary lower extremity and genital lymphoedema complicated with severe lymphorrhea. J. Plas. Recon. \& Aesth. Surg. 64 (2011), 812-815.

\section{PD-MER Pietro Giovanni di Summa,} MD, PhD

Consultant

Department of Plastic, Reconstructive and Hand Surgery, Centre Hospitalier Universitaire Vaudois (CHUV),

Lausanne, Switzerland

Rue du Bugnon 46

1011 Lausanne, Switzerland

TEL +41213142525

FAX +41213142530

Pietro.di-Summa@chuv.ch 